\title{
Technique and results in total laparoscopic radical cystectomy with sigmoidorectal pouch (Mainz pouch II) - an initial experience
}

\author{
JUNSHENG BAO, ZHONGJIN YUE, GONGJIN WU, WEI SHI and WEI WANG \\ Department of Urology, Lanzhou University Second Hospital, Lanzhou, Gansu 730030, P.R. China
}

Received April 27, 2016; Accepted December 21, 2016

DOI: $10.3892 /$ etm.2017.4163

\begin{abstract}
We used laparoscopic radical cystectomy (LRC) with urinary diversion for muscle-invasive bladder cancer. A series of total LRC is presented with Mainz pouch II after we had gained experience of nearly 200 open radical cystectomy and 23 LRC. Since from October 2010 to April 2012, we performed totally LRC in 5 patients with muscle-invasive bladder cancer with Mainz pouch II as a urinary diversion. We analyzed age, pathological staging, continence, operative time, blood loss, blood transfusion volume, and complications. The mean age of the patients was 58.8 \pm 7.6 years (range, $49-69$ years), and the follow-up time was $15.4 \pm 4.72$ months. The mean operative time was $577.0 \pm 126.4 \mathrm{~min}$ for total LRC, with a mean blood loss of $280.0 \pm 130.38 \mathrm{ml}$ and a mean hospital stay of $29.4 \pm 9.2$ days. Blood transfusion was required in two cases, and immediate and late complications were observed in 2 cases. In conclusion, total LRC with urinary diversion is feasible for the management of muscle-invasive bladder cancer, by experienced surgeons, and despite the high level of difficulty of these procedures.
\end{abstract}

\section{Introduction}

In $2013,72,570$ new cases of bladder cancer were diagnosed and 15,210 deaths occurred in the United States (1). The majority of these cancers are superficial bladder cancer at the first time of diagnosis (2), 20-40\% and infiltrative associated with a high risk of death $(3,4)$. The radical surgical removal of the bladder within urinary diversion remains the standard treatment in the management of muscle invasive or high risk bladder cancer (5).

Correspondence to: Dr Zhongjin Yue, Department of Urology, Lanzhou University Second Hospital, 80 Cuiyingmen Road, Lanzhou, Gansu 730030, P.R. China

E-mail:wjb16418@163.com

Abbreviations: LRC, laparoscopic radical cystectomy; TURBT, transurethral resection of bladder tumor; PLND, pelvic lymph node dissection; ORC, open radical cystectomy

Key words: bladder cancer, urinary diversion, Mainz pouch II
Mainz pouch II, through the detubularized sigmoid colon forms a high capacity pouch and low pressure, and was introduced by Fisch et al (6). Several studies have demonstrated that Mainz pouch II is a safe and accepted procedure of urinary diversion with high continence rate and low incidence of complications $(7,8)$.

We performed total laparoscopic radical cystectomy (LRC) with Mainz pouch II when our department had gained experience in nearly 200 open radical cystectomy (ORC) and 23 LRC.

\section{Patients and methods}

Patients. During a period of 19 months from October 2010 to April 2012, we performed total LRC in 5 patients with muscle-invasive bladder cancer with Mainz pouch II as a urinary diversion at the Department of Urology of Lanzhou University Second Hospital. The patients included 5 men with a mean age of 58.8 \pm 7.6 years (range, $49-69$ years). All patients underwent computed tomography (CT) and other analysis.

Anaesthetic risk was I in 3 patients, and II in 2 patients according to the American Society of Anesthesiologists. Only one patient underwent transurethral resection of bladder tumor (TURBT) and received preoperative chemotherapy before surgery. This study was approved by the Ethics Committee of the Second Hospital of Lanzhou University. Signed written informed consents were obtained from all participants before the study.

Surgical treatment. All patients received mechanical bowel preparation and broad-spectrum antibiotic for 3 days before the surgical procedure. The patients were given general anaesthesia, placed in a lithotomy position and set in the Trendelenburg position at 25 degrees, bilateral leg bandages were applied. An immanent bladder catheter was inserted using sterile techniques.

For placement of the camera port, first $10 \mathrm{~mm}$ trocar was placed at superior crease of the umbilicus. The pneumoperitoneum was established after insufflation of $\mathrm{CO}_{2}$ to a pressure of $14-15 \mathrm{mmHg}$ and then the second $5 \mathrm{~mm}$ trocar and third $12 \mathrm{~mm}$ trocar were placed $2 \mathrm{~cm}$ below the umbilicus in the midclavicular line on left and right sides respectively. The fourth $5 \mathrm{~mm}$ trocar was $2-3 \mathrm{~cm}$ superior and medial to anterosuperior iliac spines on the right side.

The peritoneum was opened on the furcation level plane of the common iliac vessels was dissociated the ureter until 
the bladder. The vasa deferentia and seminal vesicles were dissected, starting dissection of the space of anterior bladder wall after opening Denonvillier's fascia. At the end of this step, dissection of the pelvic fascia and puboprostatic ligament was performed. The deep dorsal vein of penis complex was controlled and then ligation was performed with 1-0 Vicryl. Vascular control of the prostatic pedicles, dorsal vein complex, urethra, prostate and bladder were then completely clipped and transected, and the bladder catheter was removed.

With the aid of unipolar forceps, $24-\mathrm{cm}$ segment of the sigmoid and rectum $8 \mathrm{~cm}$ above anus was isolated laparoscopically. Then, the dissected tissues were extracted in Endo-Pouch through rectum using sponge forceps swingingly with bladder body towards lower limbs avoiding the opened rectum rupture.

The posterior wall of the sigmoid and rectum were stitched side-to-side using 3-0 polygalactin discontinuously and then fixed to serosa by another 3-0 polygalactin, emerging from the posterior wall of the pouch. The mobilized ureters were drawn through the completed pouch plate and secured with sutures. Then a papilla was formed by suturing the mucosa over the ureters to perform an anti-reflux mechanism by laparoscopy (Fig. 1). Two F7 Double-J catheters were placed into bilateral ureter and fixed at the drainage tube after a drainage tube was inserted transanally. Eventually the anterior wall of the pouch was closed laparoscopically using 3-0 polygalactin continuously. A F22 Foley catheter was placed for drainage transurethrally.

\section{Results}

The follow-up time was 15.4 44.72 months (range 8-21 months). The urinary diversion were completed laparoscopically as planned without any intraoperative or open conversion (Fig. 2).

The histological types observed were transitional cell carcinoma in all five patients (100\%). Further, the pathological staging disclosed T2 in five $(100 \%)$ cases. Two $(40 \%)$ cases were classified as grade II, three $(60 \%)$ as grade III. Four cases were initially diagnosed, the another case was second management after cystoscopy with TURBT six months earlier.

Mean operative time was $577.0 \pm 126.4$ min ranging between 480 and $795 \mathrm{~min}$. The patients were hospitalized for a mean of $29.4 \pm 9.2$ days ranging from 21 to 43 days. Average blood loss was $280.0 \pm 130.38 \mathrm{ml}$ ranging from 100 to $400 \mathrm{ml}$. Two cases received blood transfusion (300 and $400 \mathrm{ml}$, respectively).

After removal of the rectal tube, the patients were continent, except two patients who had night incontinence, one case had initial voiding interval of 1-1.5 h for the first month post-operatively. However, three months post-operatively, the patients were continent by day and night, except one who had occasionally day incontinent when walking or coughing. The mean voided volume was on $350.0 \pm 40.82 \mathrm{ml}$ ranging between 300 and $400 \mathrm{ml}$ except one case had pyuria.

Cystography was performed post-operation to assess the configuration of the upper urinary tract and to test the antireflux procedure or leakage. As we can see, the upper urinary tract did not show any back pressure signs according to the IVU post-operatively (Figs. 3-5).

Immediate and late complications were observed in 2 cases. After Mainz II diversion one patient developed urinary

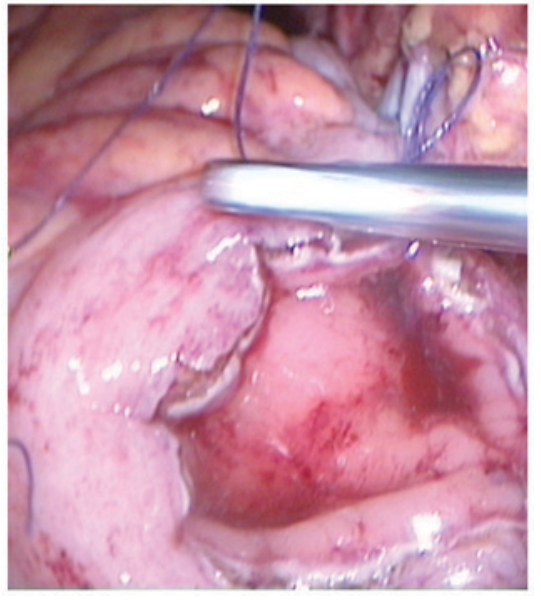

Figure 1. The sigmoid and rectum was isolated at $24-\mathrm{cm}$ segment with the aid of a unipolar forceps. The posterior wall of the sigmoid and rectum were anastomosed side-to-side and fixed to serosa by another 3-0 polygalactin, emerging from the posterior wall of the pouch.

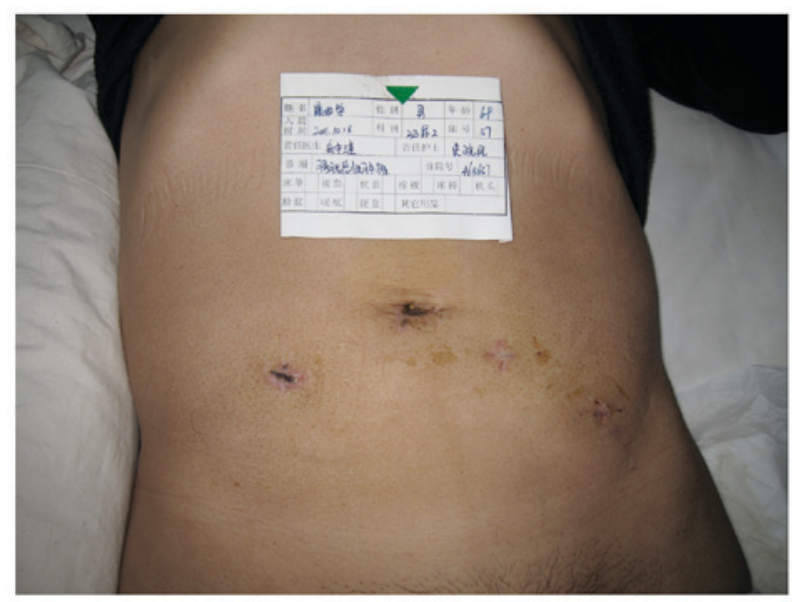

Figure 2. Abdomen after laparoscopic radical cystectomy (LRC) and Mainz pouch II diversion.

leakage seven days post-operatively and was treated with antibiotics for twelve days. One case showed fever (39 degrees) and a transanal drainage tube was placed for three days, and afterwards no other adverse events occurred.

\section{Discussion}

Radical cystectomy is the standard for muscle invasive or high risk bladder carcinoma (9). Laparoscopic treatment of infiltrative bladder tumors was introduced to be one of the surgical approaches, and developed to decrease the perioperative morbidity and trauma in the field of minimally invasive surgery. It has been reported that laparoscopic radical cystoprostatectomy with intracorporeal ileal was conducted for urinary diversion for the first time in 2000 (10). Since then, several groups reported the use of laparoscopy for radical cystectomy and indicated the safety and practicability of LRC $(11,12)$.

To our knowledge, LRC is not yet widely used in the management of infiltrative bladder tumors in many areas because of the technical difficulties of this approach, challenging urolo- 


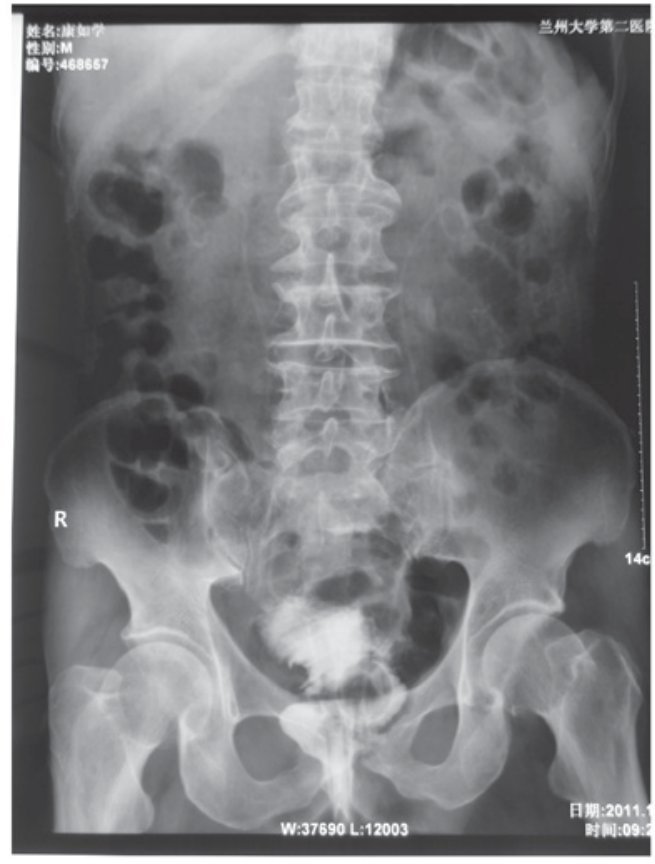

Figure 3. Seven days post-operative cystography for a 69 -year male with muscle invasive bladder cancer managed with Mainz pouch II. Urinary leakage appeared in this case.

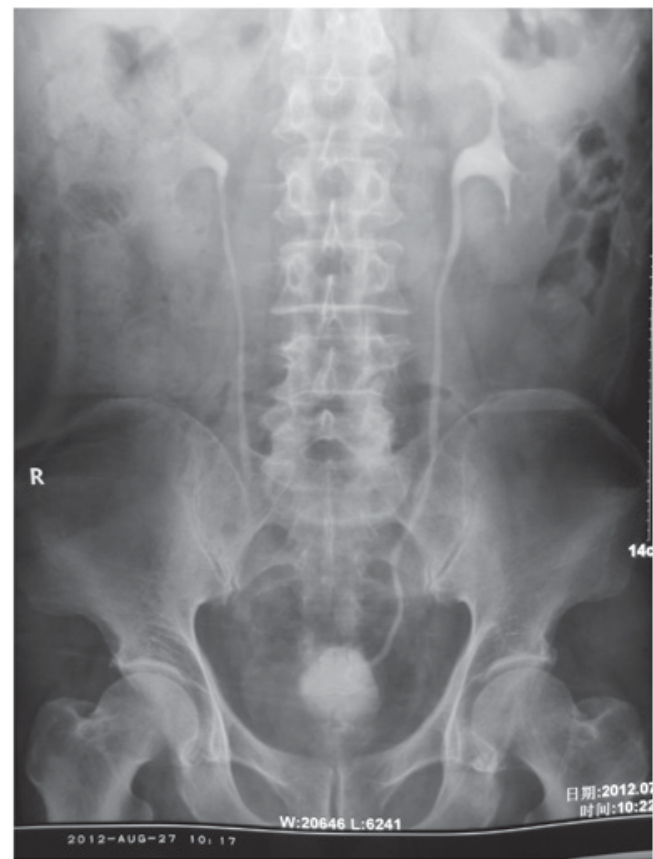

Figure 4. Upper urinary tract did not change three mouths after operation. The proximal colon did not show back wash of dye.

gist to dissect large pelvic lymph node dissection (PLND), and lengthy operative time.

Compared to ORC, LRC is conducive to expose vital tissues in the pelvic cavity (e.g., the deep dorsal vein of penis complex or sphincter urethra). LRC also leads to smaller vascular injury intraoperatively compared to PLND, fast recovery, rapid return to daily activities, and short postoperative hospital stay time.
A

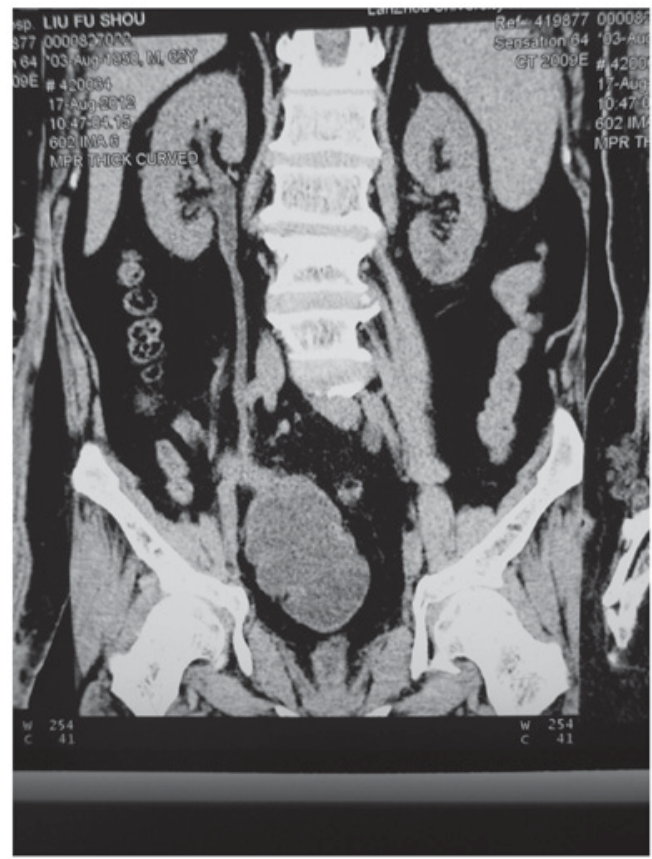

B

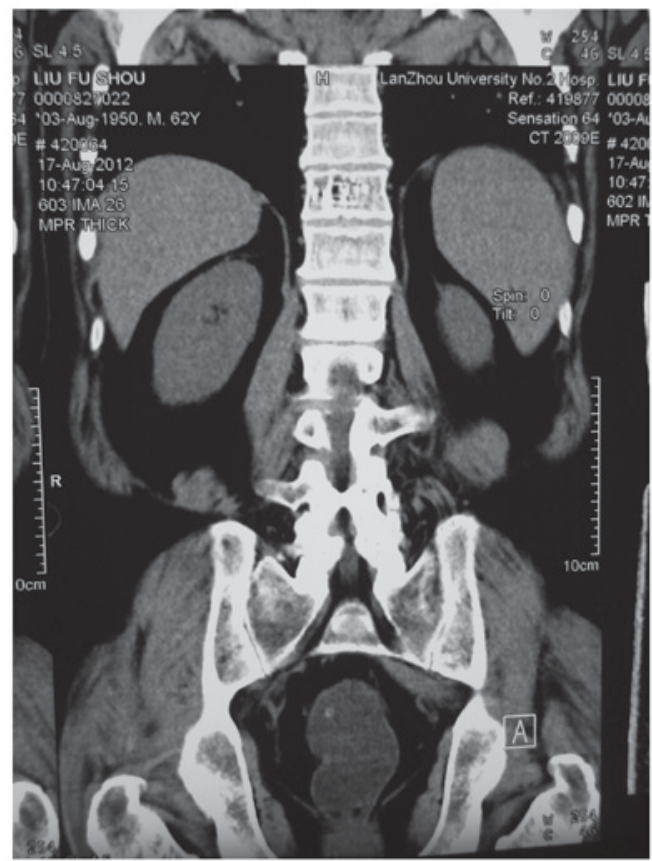

Figure 5. (A and B) Three months post-operatively computed tomography (CT) scans show the persistent wide capacity of the pouch.

Park et al (12) reported their technique and initial results in pure LRC for muscle-invasive bladder cancer. They performed ileal conduit with a $6-\mathrm{cm}$ sized midline incision extracorporeally after LRC in thirty cases. The median operative time required for extended bilateral PLVD was $527.5 \mathrm{~min}$ and no one converted to open surgery. The results indicated that LRC is a feasible management for invasive bladder cancer.

The laparoscopic group had more mean operative time, significantly less mean blood loss, analgesic requirement and transfusion requirement than in ORC group (13). The mean follow-up was 76 and $80 \%$ in LRC and ORC groups, respectively. However, the results indicated that pathological 
stage or grade, cancer-specific, and recurrence-free survival rates were not significantly different between the LRC and ORC groups in terms of 3-year overall survival (64.2 vs. 72.6, 73.0 vs. 75.3 and 70.5 vs. $72.5 \%$, respectively) (14).

Nevertheless, the data for longer follow-up of LRC in a larger cohort of patients is warranted to assess the complications rate and oncologic outcomes in the robot assisted area.

In our series, the mean operative time, hospitalization days, average blood loss, and blood transfusion rate were $577.0 \pm 126.4 \mathrm{~min}, 29.4 \pm 9.2$ days, $280.0 \pm 130.38 \mathrm{ml}$, and $40 \%$, respectively, results were similar with the study of Park et al (12). However, the operative time was longer than those in other series. The longer time maybe caused by PLND, and isolating laparoscopically the sigmoid 24-cm segment with the aid of a unipolar forceps with a more punctilious dissection.

In many series, an extended incision transperitoneal approach $\sim 6-10 \mathrm{~cm}$ was used. The scar of incision may effect the aesthetic appreciation of the abdomen, especially for women. Therefore, we isolated the sigmoid and rectum an incision instead of transperitoneal approach, which avoids increasing mental strain of patients and causing more trauma.

We also had a rate of incontinence of $20 \%$ (1 of 5 patients), which is higher than in other series. The reason may be the limited number of cases. Unfortunately, there was no female patient in our series. Thus, the difference of operative time, blood loss, continence, or complications is still lacking.

In conclusion, total LRC with urinary diversion is feasible for the management of muscle-invasive bladder cancer by experienced surgeons despite the high level of difficulty of these procedures. The key point is isolating the sigmoid and rectum a segment with the aid of a unipolar forceps, which was performed laparoscopically rather than making an extended incision by transperitoneal approach. This approach shows the benefits of laparoscopic surgery. However, more efficient performance of large samples, more rigorous long-term randomized clinical controlled trials on the outcomes and comparison with other approaches are urgently needed.

\section{References}

1. Siegel R, Naishadham D and Jemal A: Cancer statistics, 2013. CA Cancer J Clin 63: 11-30, 2013.

2. Parkin DM: The global burden of urinary bladder cancer. Scand J Urol Nephrol (Suppl): 12-20, 2008.

3. Meller AE, Nesrallah LJ, Dall'Oglio MF and Srougi M: Complications in radical cystectomy performed at a teaching hospital. Int Braz J Urol 28: 522-525, 2002.

4. Rübben H, Lutzeyer W, Fischer N, Deutz F, Lagrange W and Giani G: Natural history and treatment of low and high risk superficial bladder tumors. J Urol 139: 283-285, 1988.

5. Stenzl A, Cowan NC, De Santis M, Kuczyk MA, Merseburger AS, Ribal MJ, Sherif A and Witjes JA; European Association of Urology: Treatment of muscle-invasive and metastatic bladder cancer: update of the EAU guidelines. Actas Urol Esp 36: 449-460, 2012 (In Spanish).

6. Fisch M, Wammack R, Müller SC and Hohenfellner R: The Mainz pouch II (sigma rectum pouch). J Urol 149: 258-263, 1993.

7. Atta MA: Detubularized isolated ureterosigmoidostomy: description of a new technique and preliminary results. J Urol 156: 915-919, 1996.

8. Gilja I, Kovacić M, Radej M, Kosuta D, Bakula B and Goles L: The sigmoidorectal pouch (Mainz pouch II). Eur Urol 29: 210-215, 1996.

9. Smith JA Jr: Surgical treatment of bladder carcinoma: why is it underutilized? Cancer 104: 1563-1566, 2005.

10. Gill IS, Fergany A, Klein EA, Kaouk JH, Sung GT, Meraney AM, Savage SJ, Ulchaker JC and Novick AC: Laparoscopic radical cystoprostatectomy with ileal conduit performed completely intracorporeally: the initial 2 cases. Urology 56: 26-29, discussion 29-30, 2000.

11. Gao ZL, Fan J, Zhao JJ, Xia SJ, Shi L, Men CP, Wang H, Wang L, Yang DD, Sun DK, et al: Laparoscopic radical cystectomy with extracorporeal ileal conduit urinary diversion for treatment of Chinese bladder cancer patients. Urol Int 79: 204-209, 2007.

12. Park B, Jeong BC, Jeon SS, Lee HM, Choi HY and Seo SI: Pure laparoscopic radical cystectomy with ileal conduit: a single surgeon's mid-term outcomes. Yonsei Med J 54: 912-920, 2013.

13. Hemal AK and Kolla SB: Comparison of laparoscopic and open radical cystoprostatectomy for localized bladder cancer with 3-year oncological follow-up: a single surgeon experience. J Urol 178: 2340-2343, 2007.

14. Ha US, Kim SI, Kim SJ, Cho HJ, Hong SH, Lee JY, Kim JC, Kim SW and Hwang TK: Laparoscopic versus open radical cystectomy for the management of bladder cancer: mid-term oncological outcome. Int J Urol 17: 55-61, 2010. 